Javier Adolfo Aguirre Ramos*

\title{
ComunLAB: Análisis de caso del diseño de un laboratorio ciudadano implementado para fortalecer la sostenibilidad de colectivos culturales de la ciudad de Cali, Colombia ${ }^{1}$
}

\author{
ComunLAB: A commitment to community communication \\ in the context of socio-environmental conflicts in the Pance \\ River sub-basin in the city of Cali, Colombia
}

Cómo citar:

Aguirre, J (2019). ComunLAB: Análisis de caso del diseño de un laboratorio ciudadano implementado para fortalecer la sostenibilidad de colectivos culturales de la ciudad de Cali, Colombia. Designia , 7(2), 41-65.

\section{${ }^{1} \mathrm{El}$ artículo hace parte de la investigación doctoral} del proponente del artículo.

* Javier Adolfo Aguirre Ramos es doctor en Diseño y Creación. Profesor Investigador Universidad ICESI. E-mail: jaaguirre@icesi.edu.co ORCID: http://orcid.org/0000-0003-4344-259X

\section{Palabras clave:}

Diseño social, industria cultural, colectivos culturales, sostenibilidad, codiseño.

Key words:

Social design, cultural industries, cultural collectives, sustainability, co-design.

Recibido: 30/04/2019

Aceptado: 12/08/2018

Resumen:

El presente artículo expone los resultados de una experiencia de investigación creación, a través del estudio de caso del Laboratorio Comunitario de Medios Digitales y Contenidos Culturales-ComunLAB, laboratorio ciudadano gestado bajo las lógicas del diseño social que buscó potenciar la sostenibilidad de colectivos culturales en la ciudad de Cali, Colombia. Este estudio se estructura a partir de un análisis global, el cual se concentra en identificar las estrategias de diseño empleadas por el laboratorio, y de un análisis profundo del caso que busca dar cuenta de las contribuciones de este a la sostenibilidad de los colectivos. 


\section{Abstract:}

This article exposes the results of a research through design experience based on the study case of the "Laboratorio Comunitario de Medios Digitales y Contenidos Culturales-ComunLAB; a civic laboratory gestated under the logic of social design that sought to potentiate the sustainability of cultural collectives in the city of Cali, Colombia.

This study is structured on the basis of a global analysis, which focuses on identifying the design strategies used by the laboratory. And an in-depth analysis that aims to identify how such strategies have impacted the sustainability of the cultural collectives that were part of the laboratory.

\section{Introducción}

Tal y como lo plantea Mestres, "el diseño está en todas partes" (2012). Y es un don que desde tiempos inmemorables le ha permitido al ser humano utilizar la creatividad, las manos y los recursos disponibles para satisfacer necesidades tanto individuales como colectivas, el cual se ha materializado en una diversidad de $\operatorname{artefactos}^{1}$ que han servido y sirven a la sociedad.

Así como el ser humano, el diseño también se ha transformado. Para el siglo XVIII, con la revolución industrial, el diseño pasó de ser un generador de soluciones a un generador de necesidades; necesidades que solo podían ser satisfechas a través

${ }^{1}$ Entendido por Krippendorff (2006) como un "producto de una habilidad humana entrenada" del consumo de productos, lo que permitiría la entrada de un sistema en el que la sociedad trabaja y vive para alcanzar un ideal de identidad basada en la capacidad de consumo. En este contexto, el diseño se transformó en uno de los principales aliados del sistema capitalista. 
Sin embargo, en las décadas de los setenta y ochenta del siglo XX surgió en el panorama de la disciplina un llamado que defendía la idea de que el diseño debería darse dentro de un marco de acciones éticas en el que fuera consciente de sus implicaciones sociales y ambientales. Las discusiones, cuestionamientos y replanteamientos acerca del quehacer del diseño generaron profundos cambios en sus prácticas y campos de acción. Tales transformaciones han posicionado al diseño como una disciplina centrada en lo humano y enfocada en actuar sobre diversas problemáticas que aquejan a la sociedad, cuestionando su tradicional función al servicio del consumo y poniendo en evidencia la necesidad de fortalecer el compromiso social del diseño y de los diseñadores (Aguirre y Cifuentes, 2018).

De este modo, en aras de enfrentar los complejos desafíos que afectan a la sociedad actual, la disciplina cuestionó sus paradigmas dominantes (Aguirre y Cifuentes, 2018). Así, el diseño se reconstruyó dentro de un nuevo modelo de paradigmas que sirvió como herramienta de cambio, generador de solución antes que de necesidad y, al mismo tiempo, ayudó a la construcción de un nuevo quehacer en el diseño, en donde este fuera planeado y ejecutado con la comunidad misma. Surge entonces el diseño social, un campo de acción de la disciplina capaz de realizar valiosos aportes, y que de manera amplia se entiende como la práctica del diseño orientada hacia fines colectivos y sociales, en lugar de perseguir objetivos esencialmente comerciales o de consumo (Armstrong et. al, 2014).

De este cambio de paradigma se desprenden nuevas formas de llevar a cabo el proceso de diseño, que permiten entender, comprender e identificar lo que es el diseño social. Una de ellas es el de codiseño. "Por codiseño, nos referimos a la creatividad colectiva que se aplica en un proceso de diseño (...) codiseño es una instancia específica de la cocreación" (Sandres y Simons, 2009, p.1). De esta manera, tanto el proceso de diseño como su materialización es pensado, hablado y realizado por la comunidad y el diseñador. Por ende, lo que intenta el codiseño es lograr que todo lo que se realice esté sustentado bajo las lógicas de la comunidad misma. Para Sanders (2006), la participación activa de las personas en el proceso de diseño asegura que los sistemas, productos o servicios que se diseñen satisfagan plenamente la necesidad de las personas. Por ello, es de suma importancia que ellas participen, en la medida de lo posible, en todo el proceso.

Siguiendo con lo anterior, se hace relevante comprender las estrategias necesarias que se requieren o que pueden ser utilizadas en el proceso de diseño social. Meroni (2008, p. 62) sugiere que la misma capacidad estratégica que el diseño aplica cuando trabaja para solucionar un problema en el ámbito industrial puede ser utilizado para solucionar problemas sociales. En este contexto, contar con un enfoque estratégico 
${ }^{2}$ El proyecto Industrias

Culturales de Cali pretende convertir a la ciudad en

referente latinoamericano por su potencial cultural, artístico y empresarial. Esta iniciativa, busca impulsar el desarrollo económico y social de la capital del Valle a través del fomento del talento creativo, el fortalecimiento de redes en el proceso de diseño social permite generar sistemas, productos o servicios que benefician a la comunidad, mejorando su calidad de vida, manteniendo y desarrollando su propia identidad.

Por su parte, Holston (2011) propone cuatro principios que orientan el diseño estratégico: capacidad para solucionar problemas complejos, trabajar colaborativamente, diseñar en contextos diferentes a un despacho de diseño y realizar acciones responsables asociadas a las seriedad y el compromiso que el diseñador debe asumir con la comunidad.

Asimismo, el diseño social demanda de los diseñadores mucho más que solo un proceso creativo. Cross plantea que enfrentarse a un problema de diseño implica no solo desarrollar una serie de actividades para alcanzar la solución, sino que es necesario “(...) reflexionar un poco sobre la forma en que se está abordando el problema, lo cual requiere cierto pensamiento estratégico acerca del proceso de diseño" (Cross, 1999, p.63). Por ello, es importante que en este contexto el diseño sea no solo una herramienta de creación, sino también de reflexión, que se cuestione acerca de las problemáticas sociales y de las formas en las que pueden ser abordadas.

La recomendación del autor para alcanzar los objetivos tratados en un proyecto de diseño son "diseñar una estrategia que responda al problema o a la situación particular, mantener flexible la estrategia y revisar periódicamente su eficacia durante el proyecto de diseño" (1999, p.187). Por lo tanto, cualquier proceso de diseño dentro de un contexto social debe construirse por y para las problemáticas de la comunidad, teniendo en cuenta las variables del tiempo y espacio que pueden llevar a reorientar el proceso varias veces, ya que dentro de este proceso, tanto la realidad del diseño como la de la comunidad puede cambiar frente a las problemáticas y necesidades que se presenten.

Para lograr el proceso de diseño social se debe comprender mejor los problemas sociales, crear escenarios y visiones compartidas con la comunidad, facilitar acceso al conocimiento, aportar en el desarrollo de capacidades, enfrentar el contexto cambiante y la incertidumbre, y emplear métodos y herramientas que le permitan alcanzar los objetivos sociales. Además, debe permitir alinear el resultado con los deseos y objetivos de la comunidad.

En el marco de una investigación desarrollada como parte del proceso de formación doctoral del proponente del artículo, se realizó un estudio de caso sobre un laboratorio ciudadano que sirvió como medio para generar un espacio de encuentro y diálogo entre el Proyecto de Industrias Culturales de Cali (PRIC) ${ }^{2}$ y colectivos culturales ${ }^{3}$ de la misma. 


\section{Consideraciones metodológicas}

El Laboratorio Comunitario de Medios Digitales y Contenidos CulturalesComunLAB ${ }^{4}$ es un laboratorio ciudadano que buscó fortalecer los procesos agenciados por los colectivos culturales de la ciudad de Cali a través de la promoción de dinámicas de experimentación que fomentan la cultura libre, la colaboración y los modelos de aprendizaje colaborativo.

Como base para la construcción de este estudio de caso se establecieron los siguientes objetivos que direccionaron la construcción de su análisis:

a) Identificar estrategias y tácticas de diseño utilizadas en el Laboratorio Comunitario de Medios Digitales y Contenidos Culturales-ComunLAB.

b) Analizar los beneficios generados por el Laboratorio Comunitario de Medios Digitales y Contenidos Culturales-ComunLAB, en clave de los aportes a la sostenibilidad de los colectivos y su relación con las estrategias de diseño implementadas.

La investigación acogió los postulados de Gumucio (2005), quien plantea que son tres los tipos de sostenibilidad que afectan a una organización comunitaria: la sostenibilidad económica, social e institucional. "Sin un equilibrio entre los tres factores, la sostenibilidad a mediano y largo plazo es imposible" (Gumucio, 2005, p.8).

La selección de esta experiencia como caso de estudio tiene como sustento la articulación de variables como diseño social y sostenibilidad; a lo que se le suman las características del laboratorio, sus antecedentes, trayectoria, y la cercanía de quien lideró la investigación. De esta manera, el ComunLAB busca promover dinámicas 
de experimentación innovadoras que fomentan la cultura libre, la apropiación de tecnologías y los modelos de aprendizaje colaborativo. La iniciativa se inscribe en las dinámicas de los llamados laboratorios ciudadanos, en tanto espacios en los que...

\begin{abstract}
Personas con distintos conocimientos y diferentes grados de especialización se reúnen para desarrollar proyectos juntos. [Son] espacios que exploran las formas de experimentación y aprendizaje colaborativo [...] Estos proyectos trabajados y generados en laboratorios ciudadanos tienen la característica de buscar una transformación social, que contribuya al desarrollo cultural, social y económico (Proyecto Ciudadanía 2.0 de la Secretaría General Iberoamericana-SEGIB, 2014).
\end{abstract}

Es de especial importancia el interés de este laboratorio por los colectivos culturales de la ciudad, que representan su motor y horizonte de acción. Adicionalmente, se consideró pensar su orientación, por un lado, respecto a las dinámicas que toman lugar en la ciudad y, por el otro, a la pregunta transversal por la sostenibilidad de los colectivos que lo conforman. Igualmente, el laboratorio se ha constituido como un espacio en el que convergen diversas perspectivas y que propicia el encuentro y la discusión.

Finalmente, con relación a la noción de diseño social, se suma a los elementos enunciados que su existencia no se orienta ni está en función de prácticas comerciales y/o empresariales. Por el contrario, busca potenciar las iniciativas de los colectivos, tejer redes entre quienes participan, y propender por actuar en beneficio de los colectivos que allí se convocan cada año, los cuales tienen el potencial de ser multiplicadores de saberes y experiencias con otros colectivos y organizaciones, no solo de la ciudad sino del país.

A esta investigación interesa, más que los aspectos formales y funcionales, el "entender el diseño como plan, como proceso creativo de reflexión en acción, a través del intentar resolver retos desde la empatía, la contextualización y el prototipado" (Mestres, 2012, p.143). Desde esa mirada, ComunLAB hace parte de un diseño que vincula elementos centrales para resolver los intereses que orientan esta investigación, entre ellos están: 1) centra su atención en los colectivos culturales de la ciudad y su sostenibilidad; 2) propone un diálogo continuo con las dinámicas y las problemáticas de la ciudad; 3) posee capacidad de articulación con diversos actores sociales. 
Para el desarrollo del caso se utilizaron diferentes metodologías para recabar los datos analizados. En primera medida, se recurrió al material de archivo vinculado a las cinco versiones del laboratorio realizadas hasta el momento. De este fueron de utilidad los informes de gestión de cada una de las versiones, al igual que los vídeos y los audios que conforman las memorias del evento. Para la recolección de datos de fuentes primarias se realizó un grupo focal con 17 integrantes de 8 colectivos de la ciudad que han estado vinculados al proceso, tanto en su fase de construcción como en calidad de asistentes al laboratorio. Adicionalmente, se diseñó una entrevista semiestructurada que fue aplicada a actores que han sido relevantes para la construcción del laboratorio: el director y tres de sus coordinadoras.

Los datos recabados durante esta etapa del estudio de caso fueron analizados bajo los dos objetivos planteados inicialmente. En primer lugar, con el propósito de detallar el proceso de construcción del laboratorio, en clave de las estrategias y las tácticas implementadas para su desarrollo, tanto de carácter conceptual como de las actividades que toman lugar anualmente. Paralelamente, se orientó a la identificación de los beneficios que la plataforma ha podido generar, junto a los aportes para la sostenibilidad de los colectivos. 
Una vez esbozada la ruta metodológica utilizada para el caso en particular, se procedió a exponer el contexto en el que surge y se desarrolla ComunLAB. El laboratorio inició en 2011 y terminó en 2015, años en los que contó con cinco versiones en las cuales se generaron distintas dinámicas de intercambio de saberes cooperativos y abiertos en la ciudad de Cali. Se trató de un espacio anual en el que, durante varios días, tal como lo hacen los laboratorios ciudadanos, se construyó a partir de los aportes de sus participantes y del apoyo en una red distribuida de personas y otras organizaciones.

Igualmente, ComunLAB promovió la participación activa de una red colaborativa conformada por comunidades, colectivos, instituciones, gobierno local, ONG, entre otros. El laboratorio visibilizó el trabajo de los colectivos culturales, propició colaboraciones y promovió la apropiación de tecnologías digitales como medio para transformar los contextos en los que se inscribían sus prácticas. Sus cinco versiones fueron posibles gracias a la participación activa de individuos, colectivos e instituciones.

Año tras año, el laboratorio fue un espacio para la experimentación mediante modelos colaborativos y horizontales - intercambios libres de saberes, espacios de difusión y creaciones compartidas - encaminados al fortalecimiento del quehacer colectivo y los procesos sociales y culturales existentes, propendiendo así por la consolidación de una plataforma de soporte para que diferentes actores sociales generaran y compartieran conocimientos de manera abierta, enfocados en plantear propuestas en beneficio de los procesos sociales y culturales existentes en la ciudad.

La estructura con la que funcionó el laboratorio cambió paulatinamente conforme las dinámicas de cada versión y los aportes que, año tras año, hicieron quienes participaron. Al respecto, se debe considerar que este tipo de proyectos son reflexivos e "incorpora[n] a los ciudadanos en este proceso y que, por tanto, aprende[n] y evoluciona[n] constantemente" (Proyecto Ciudadanía 2.0 de la Secretaría General Iberoamericana-SEGIB, 2014, p.6).

No obstante, siempre se mantuvo la intención transversal de conocer a los colectivos, que se conocieran entre ellos y que la institucionalidad los reconociera. Igualmente, sirvió como un espacio para aprender, compartir, discutir sus problemáticas y temas de interés común. 
Año tras año la realización del laboratorio comprendió las siguientes etapas, que contemplaron varias actividades:

\section{Etapa 1: Planeación}

En esta etapa se coordina el plan de trabajo que da forma al laboratorio en cada versión. Se convoca a quienes han participado en ocasiones anteriores para vincularse al diseño del laboratorio, los colectivos, organizaciones y personas interesadas. De manera que se da una participación activa durante todas las etapas del laboratorio, gracias a la cual se definen los contenidos de los talleres-intercambios y las actividades a realizar, para así consolidar la agenda del evento. Se establecen las necesidades logísticas para su desarrollo y se despliega el plan de promoción y divulgación para convocar a los asistentes.

\section{Etapa 2: Ejecución}

Las actividades del laboratorio se estructuran en cinco ejes, inspirados en el proceso de diseño:

- Conocer: Momento en el que cada uno de los asistentes se presenta y se ponen en común las problemáticas y los interrogantes que se trabajarán durante el encuentro. Forman parte de este eje la socialización y la mesa de discusión.

- Idear: Aquí toman lugar los talleres gratuitos e intercambios de saberes, que apuntan a pensarse cómo desde el uso de la tecnología se puede intervenir sobre las problemáticas relacionadas con la temática del laboratorio.

- Prototipar: En su mayoría, de cada taller se derivan prototipos experimentales que buscan dar solución a las problemáticas planteadas.

- Probar: Cada taller reflexiona sobre su prototipo y se discuten colectivamente las posibilidades e implicaciones que este puede llegar a tener en sus contextos y comunidades.

- Compartir: Al final se ponen en común los resultados de los talleres, intercambios y actividades; se plantean conclusiones y nuevos interrogantes. Adicionalmente, en este eje se encuentran las actividades artísticas como muestras y conciertos, productos, en muchos casos, de los mismos talleres e intercambios. 


\section{Etapa 3: Memorias y reflexión}

Una vez culminado el encuentro, cada año se hace un balance del laboratorio, se identifican sus debilidades y fortalezas, y se plantean oportunidades de mejora para las futuras versiones.

Una vez expuestas las consideraciones generales de la realización y las versiones del laboratorio, se presenta el análisis global y el análisis profundo del caso. El primero, se concentra en las estrategias de diseño de las que ha hecho uso el laboratorio. El segundo, plantea las contribuciones de este espacio a la sostenibilidad de los colectivos que en él han participado.

\section{Análisis global}

Esta primera parte del análisis de caso corresponde a la identificación de las estrategias de diseño que utilizó el laboratorio. Para ello, se toman como punto de partida los hallazgos sobre las estrategias de diseño que se implementan en los procesos de diseño social y se identifican cuáles están presentes en ComunLAB, cómo se desarrollan y qué particularidades adquieren. Así pues, son tres los ejes sobre los que se identifican las estrategias y tácticas: 1) estrategia utilizada en el proceso de codiseño; 2) estrategia utilizada para generar dinámicas de colaboración y trabajo en equipo; 3) estrategia de lugar y condiciones de trabajo.

\section{Estrategia definida para el proceso de codiseño}

La estrategia planteada para incidir sobre las los cambios de la sostenibilidad social, institucional y económica a través de ComunLAB, no siguió inicialmente un plan establecido. Siguiendo a Cross (1999, p.175), "el no tener un plan aparente de acción puede ser también una estrategia. Podría denominarse una estrategia de búsqueda al azar (divergente) y ser apropiada en situaciones de diseño novedosas de gran incertidumbre, donde se está haciendo la búsqueda más amplia posible de soluciones". 
Aunque ComunLAB se planteó en un pincipio como una acción que buscó generar un espacio de encuentro entre los colectivos culturales y el PRIC para discutir sobre la industria cultural, el proceso de codiseño del laboratorio se construyó versión a versión según las particularidades que trajo consigo la temática y la consecución de recursos para cada año. Sin embargo, para su análisis se siguió la síntesis del proceso de codiseño (adaptación proceso de codiseño propuesto por Sanders y Stappers, 2008), el cual se compone de las siguientes etapas: (Ver Gráfico 1).

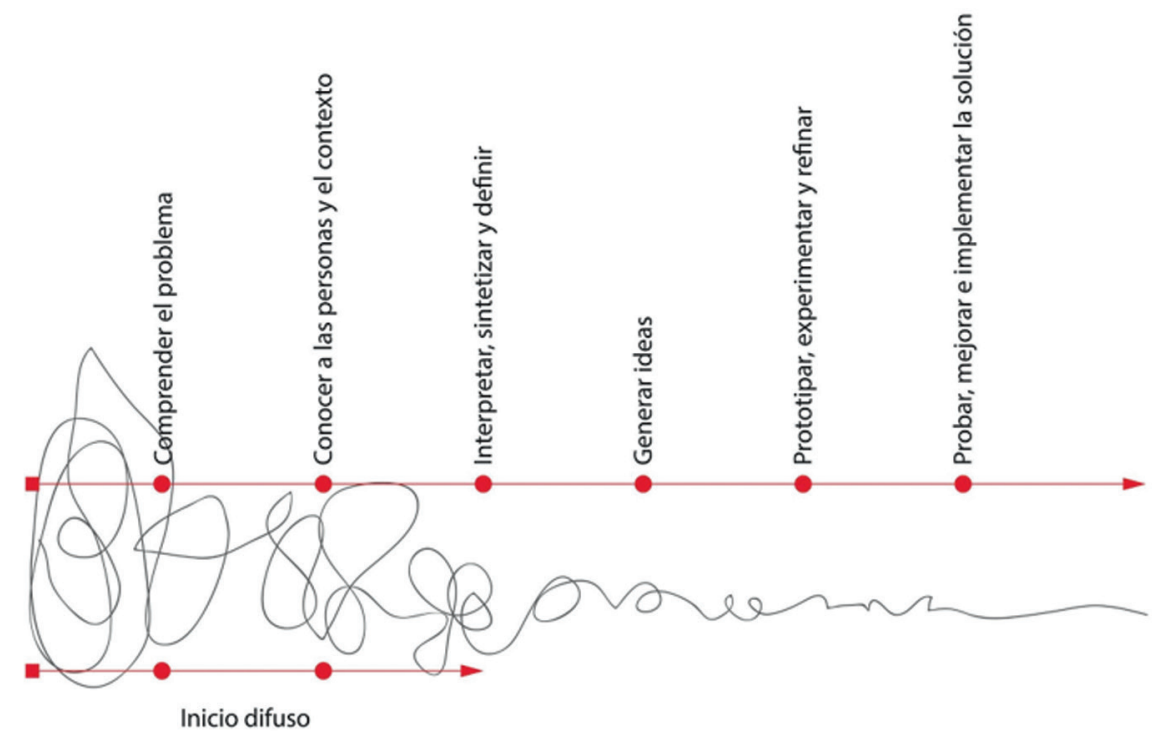

Gráfico 1. Proceso de codiseño. Fuente: Adaptación modelo de codiseño propuesto por Sanders y Stappers (2008). 
En los subtítulos subsiguientes se analizan las etapas de la estrategia y las tácticas que se han utilizado durante el desarrollo del laboratorio.

\section{Comprender el problema}

Dentro de las acciones que implementó el laboratorio para comprender el problema de la sostenibilidad - que a su vez resulta ser lo que alienta la creación del ComunLAB - se identifica, en primer lugar, acercarse a los colectivos de la ciudad y buscar entender sus dinámicas y prácticas. Entre las acciones para comprender el problema se encuentra el acercamiento de los directores y coordinadores a diferentes colectivos, a propósito de su asistencia a otros eventos como LabSurLab ${ }^{5}$, pero también por conexiones con dichas organizaciones que anteceden a la creación del laboratorio.

En cuanto a la búsqueda de diferentes perspectivas sobre el problema, quienes estaban a la cabeza del laboratorio desde sus inicios buscaron entender las diferentes perspectivas sobre los procesos que impulsan los colectivos y sus lógicas. Allí, aquello que catapultó la creación de una propuesta para el Proyecto de Industrias Culturales fue encontrar concepciones disidentes entre lo que los colectivos realizaban y la manera en que estos se definen, en contraposición a la postura del PRIC que, desde su mirada empresarial, comprendía a los colectivos como emprendimientos.

\begin{abstract}
5 "LabSurLab es una plataforma de acción educativa que se articula en torno a las nociones de arte, ciencia, tecnología y
\end{abstract}

comunidades. Es una red de iniciativas que conforman: hackbals, hackerspaces, medialabs y todo tipo de laboratorios y colectivos biopolíticos operando desde y para territorios [del sur] de América, buscando desde la experimentación y creación lograr sus propios espacios de acción y representación". Recuperado de: http://proyectoidis.org/labsurlab/
Adicionalmente, como procedimiento que ha facilitado la construcción del laboratorio y la comprensión de problemáticas que atraviesan los sentidos de este, tal como lo es la sostenibilidad, se encuentra el continuo contacto y conversación con los colectivos desde una actitud de escucha y construcción a partir del diálogo.

Otro de los procedimientos que han ayudado a comprender el problema es la investigación Estudio de caracterización del perfil de los innovadores sociales de la red de medios culturales de la ciudad de Cali, llevada a cabo en 2013. Este estudio realizó una caracterización de algunos colectivos de la ciudad, en clave de temas como la innovación social, la apropiación tecnológica y la sostenibilidad de estas organizaciones. Entre las estrategias ejecutadas, la investigación estableció una orientación específica a comprender la sostenibilidad de los colectivos.

Todas estas tácticas han tenido la intención de poder ampliar el conocimiento general sobre los colectivos, al igual que indagar por la sostenibilidad de estos y sus trayectorias; partiendo siempre de dos postulados: 1) identificar el problema desde la perspectiva propia de los actores que hacen parte de los colectivos y 2) buscar alinearse con las iniciativas que anteceden la creación del laboratorio. 


\section{Conocer a las personas y el contexto}

Además de las acciones mencionadas en el punto anterior, que sin duda guardan relación directa con esta etapa del proceso de codiseño, es preciso añadir que el proceso de construcción de ComunLAB no puede ser concebido como un proceso en el que las etapas se anteceden unas a otras. De hecho, etapas como esta (conocer a las personas y el contexto) no se agotan en el proceso de codiseño, sino que continúan ocurriendo año tras año, e inclusive se alimentan y toman lugar en la misma puesta en marcha de cada una de las versiones que se realizan del laboratorio.

Lo anterior, expresado en términos de tácticas, se traduce en varias acciones. Por un lado, aquellas que se han realizado durante el laboratorio, como lo son la creación de memorias (radiofónicas, audiovisuales y fotográficas), entrevistas y testimonios; además de aquellos productos de los talleres realizados, resultado de la creación colectiva. Por otro lado, está la identificación de líderes y actores clave para alimentar las prácticas del laboratorio. Esta decisión pasa por la misma experiencia que han tenido tanto los colectivos como las instituciones con las diferentes versiones del espacio y de cómo este se ha ido proyectando.

En este sentido, una decisión clave dentro de esta etapa fue vincular a la coordinación del laboratorio a una integrante de un colectivo que había participado activamente en versiones anteriores. Ella estuvo encargada de la estructuración y mediación en la propuesta pedagógica a desarrollarse en los diferentes talleres e intercambios de saberes durante el encuentro, lo que incidió favorablemente en el laboratorio en la medida en que establece una comunicación más cercana con los colectivos y los procesos que estos realizaban. 
A lo largo de las cinco versiones de ComunLAB, se buscó que la participación de los colectivos tuviera un papel preponderante en el establecimiento de las necesidades y propuestas. Es por esto que para la realización de cada versión fueron esenciales las reuniones con los líderes de los colectivos, en las que se pensó y soñó colectivamente cada laboratorio. La construcción de una visión de conjunto y la posibilidad de potenciar las prácticas y relaciones que construyeron los colectivos mucho antes del laboratorio constituyó un componente que le dió soporte a lo propuesto por el ComunLAB.

Lo anterior se podría sintetizar en la firme intención del laboratorio de ser pertinente para los colectivos, las dinámicas de la ciudad y para ejercer un diálogo constante con los contextos en los que se enfocaban las prácticas de los colectivos, junto a la idea de una construcción participativa y horizontal. Para ello, a partir de la segunda versión se implementaron convocatorias en las comunidades a través de los colectivos, con la intención de identificar a aquellas interesadas en algún tipo de apropiación tecnológica. Una vez identificada la comunidad y la problemática, se invitaba a otros colectivos a cooperar en un proceso de intercambio mutuo de conocimientos. En estos intercambios no solo podían participar los miembros de la comunidad beneficiada, sino cualquier persona interesada en aprender sobre esa tecnología.

\section{Generar ideas}

Los métodos que predominaron en la generación de ideas fueron aquellos que permitieron la participación de diferentes actores en cada planeación de los laboratorios, a lo que se suma la experiencia que año tras año generó la posibilidad de reinventar este espacio. La experiencia y el conocimiento de los mismos colectivos sobre las comunidades fue la mayor fuente para la generación de ideas en el proceso de codiseño.

Una fase fundamental en los procesos de codiseño es conocer el contexto en el que van a desarrollarse. Este conocimiento se logra a partir de un trabajo de acercamiento y mapeado que se realiza con ciertas características, considerando el tipo de proceso con que se trate. Así pues, para este proceso se emplearon técnicas de mapeo - fundamentales en los procesos colaborativos - para identificar las necesidades de las comunidades y la manera en que ComunLAB realizaría su aporte. 
La forma de administrar y ejecutar los presupuestos gestionados para el desarrollo de las actividades en cada laboratorio - con cantidades variables entre versiones trajo consigo ejercicios creativos. Por un lado, fueron optimizados, y por el otro, resultaron de mayor beneficio para los colectivos y los participantes de cada versión. Esto implicó repensar las maneras en que generalmente se organizan los presupuestos para eventos y los compromisos que se establecen con quienes aportan recursos. Igualmente, los resultados de los laboratorios y la motivación que generó en los participantes permitió, inclusive, que la generación de ideas no se agotara en la etapa de planeación anual, sino que estuviese presente en el quehacer cotidiano de los colectivos.

\section{Prototipar, experimentar y refinar / Probar, mejorar e implementar la solución}

Hablar del prototipado del laboratorio supone referirse al mismo proceso de desarrollo de cada versión, puesto que en sí mismos son prototipos que se fueron ajustando al implementarse, entregando así nuevo conocimiento y experiencia que enriqueció las siguientes versiones. Precisamente, esto ocurrió con la estructura que hasta la última versión estuvo presente: conocer, idear, prototipar, probar, compartir. Lo cual fue el resultado de comprender los sentidos del laboratorio, al igual que las necesidades y motivaciones de quienes asistieron.

Para la primera versión, dicha estructura no estaba muy clara, pero a medida que se fueron dando las actividades en ese primer experimento, la misma estructura fue emergiendo y se fue atizando a lo largo de las siguientes versiones. Otro elemento que permitió asegurar cambios y fortalecimiento del laboratorio guarda relación con la participación continua de colectivos o algunos de sus integrantes en la planeación del mismo. 
Para que la comunidad afronte la tarea común tiene que trabajar en equipo. Trabajar en equipo es colaborar. Y cualquier proceso colaborativo tiene que dotarse de órganos y herramientas para coordinarlo, facilitarlo y hacerlo más abierto y democrático (Mestres, 2012, p.137). Dentro de la estrategia definida para este propósito, el proceso de codiseño de ComunLAB poseía varios tipos de tácticas: 1) integración de equipos de trabajo diverso en conocimiento y experiencia; 2) fomentar la participación activa de los colectivos y comunidad; 3) creación colectiva; y 4) generación de confianza.

ComunLAB, como un espacio de interlocución entre diversos actores sociales, contó con el apoyo de personas e instituciones que permitieron articular diferentes miradas en torno a las prácticas de los colectivos y la tecnología. Además de esto, el laboratorio le apostó a los espacios de aprendizaje horizontales, partiendo de que hay una gran riqueza en los saberes y aportes de cada persona, indistintamente de su contexto, edad, formación.

Lo anterior se dio especialmente en la realización de los talleres, porque más allá de pensar en un saber experto que imparte algún conocimiento, los encuentros se dieron en clave de intercambios donde todo el que asistía aprendía. El trabajo en red que caracteriza el quehacer de los colectivos tiene consigo "conexiones que a lo largo de sus diferentes trayectorias han ido generando (...) [las cuales han permitido que se amplíe el trabajo colaborativo y la participación, en tanto el] modelo de trabajo [es] proclive al cambio constante, la reflexión y la mejor concreción de las acciones buscadas" (Mestres, 2012, p.27).

Otro aspecto que incide en que las dinámicas de colaboración se den y tengan continuidad es la generación de confianza. En un principio resultó complejo, pues la relación con las instituciones, generalmente, es crítica. No obstante, la presencia de integrantes de los colectivos en el equipo organizador fue un elemento importante para promover la vinculación de otros colectivos y recibir sus aportes.

Finalmente, se puede decir que lo que terminó incentivando el trabajo colaborativo, al igual que la adhesión de colectivos y participantes, fueron los resultados generados por el laboratorio, en tanto fue pertinente y cumplió con las expectativas de muchos frente a las actividades desarrolladas. 


\section{Estrategia de lugar y condiciones de trabajo}

El ComunLAB proporcionó un espacio de encuentro entre los colectivos en el que se propició la creación colectiva, el trabajo en red, la apropiación de tecnologías, etc. A lo largo del desarrollo de cada versión se buscó que los espacios en los que se realizara el encuentro fueran accesibles al público y estuvieran alejados de la formalidad que, a veces, imponen los espacios institucionales y académicos.

Es por ello que, en varias ocasiones, algunas actividades del laboratorio se realizaron en los lugares donde se encontraban las comunidades. Igualmente, se exploraron espacios públicos de la ciudad, pero también lugares que parecían muy institucionales y que fueron resignificados en el desarrollo de la propuesta de ComunLAB, como ocurrió, por ejemplo, con el Hotel Aristi ${ }^{6}$, en sus primeras versiones. Asegurar la gratuidad de las actividades y construir una agenda amplia fue también una de las tácticas para hacer de este espacio anual un intercambio ameno en el que los participantes se sintieran cómodos.

\section{Análisis profundo}

A partir de la propuesta de Gumucio (2005) sobre la sostenibilidad de experiencias similares a los colectivos culturales en Latinoamérica, este momento del análisis expone los aportes que ha hecho el laboratorio para fortalecer la sostenibilidad de los colectivos que han participado en él. Así pues, se dará cuenta de cómo las

${ }^{6}$ Emblemático hotel ubicado en el centro histórico de la ciudad de Cali, construido por el empresario Adolfo Aristizábal. Inaugurado en 1951, es contiguo al también histórico Teatro Aristi, ambos considerados patrimonio urbano-arquitectónico de la ciudad, por su estilo art decó (Cruz Hoyos, 2016). estrategias implementadas han aportado a la sostenibilidad social, institucional y económica de estas organizaciones.

\section{Aportes generales}

Para comenzar, es preciso señalar que en la ciudad existen pocos espacios en los que se puede afianzar el encuentro entre los colectivos que realizan diferentes prácticas sociales, políticas y culturales. Para algunos de los colectivos, el laboratorio propone ser un "espacio más horizontal de discusión, debate y agitación cultural para beneficio de las comunidades de nuestra ciudad, tanto en la parte urbana como rural" (Colectivo Satélite Sursystem, 2013). 
Además, permite "reconocer que en la ciudad emergen y se están gestando proyectos. Que hay otra variedad de oferta y otra variedad de temáticas, y otras nuevas propuestas de las que comúnmente conocemos" (Coordinadora del ComunLAB 2011). Sobre la propuesta del laboratorio, los colectivos que han participado coinciden en que les ha permitido reivindicar y legitimar sus prácticas, que llevan implementando desde hace mucho tiempo. A esto se le suma que permite acceder al conocimiento fuera de los espacios institucionales y académicos.

Otro componente importante que ha resultado beneficioso para los colectivos tiene que ver con el eje transversal que impulsa el laboratorio: la apropiación de tecnologías, pues "asistimos a un momento de creación de nuevas sensibilidades a través de las tecnologías, la gran riqueza [de ComunLAB] es el encuentro entre distintas formas de asumir la tecnología para la ciudad" (Colectivo Satélite Sursystem, 2013).

ComunLAB entendió la tecnología como el "conjunto de teorías y de técnicas que permiten el aprovechamiento práctico del conocimiento científico" (RAE) y desde esta perspectiva promovió la apropiación de tecnologías sociales a través del intercambio libre de conocimientos entre individuos, colectivos, comunidad e instituciones (Chui et al. 2012). La apropiación de estas tecnologías mejoraron la capacidad de gestión, producción y comunicación de los colectivos y de las comunidades en las que tomó lugar ComunLAB. 


\section{Sostenibilidad social}

Acorde con Gumucio (2005), la sostenibilidad social toma lugar cuando las comunidades se apropian de los procesos culturales comunitarios. Para ello resultan importantes elementos como la afinidad con el proyecto político, la participación activa de la comunidad durante los procesos, la pertinencia dentro del contexto en el que toma lugar, el reconocimiento y respeto por los bienes comunes y la capacidad del proceso para contribuir al logro de los objetivos comunitarios.

ComunLAB hace suya "la idea de lo cercano, de lo local vinculado con el mundo; las nociones de proximidad, de colaboración, de red y procomún" (Mestres, 2012, p.32). En esta medida, propicia que los colectivos "se encuentren con otros que ni siquiera conocían y están trabajando en lo mismo, o sea que es un ejercicio de reconocimiento de las prácticas, los saberes y eso implica un ejercicio de ciudadanía cultural" (Colectivo Satélite Sursystem, 2013).

El valor del encuentro ha redundado en sus prácticas, en tanto "se replican los conocimientos, se transmiten, se reorientan o se resignifican y surgen nuevos eventos, nuevos procesos, nuevos colectivos, nuevas hibridaciones" (Colectivo Satélite Sursystem, 2013). En términos de los proyectos políticos que orientan a los colectivos, estos reconocen que este es un espacio importante "para el consenso y el disenso en torno a la tecnología y al proyecto político de construcción de la ciudad" (Colectivo Satélite Sursystem, 2013).

Al pensar esta dimensión de la sostenibilidad de los colectivos, la apropiación de tecnologías como elemento central del laboratorio juega un papel muy importante en los aportes que propicia el ComunLAB, en tanto propende por la democratización de las tecnologías. De otro lado, las actividades propuestas por el laboratorio han permitido estrechar, en algunos casos, los vínculos entre los colectivos y las comunidades a las que estos dirigen su trabajo. Como ocurrió, por ejemplo, con el colectivo Noís Radio, que pudo reactivar sus relaciones con un colegio con el que ya habían adelantado un proceso, a partir de la realización de un taller de radio con algunos estudiantes interesados en desarrollar un proyecto radiofónico para tal institución. 
En cuanto a la sostenibilidad social, también resulta interesante la posibilidad que ha brindado el ComunLAB de generar y/o ampliar otros procesos a partir de aquello que se ha aprendido y construido en este espacio. Lo anterior ha ocurrido, por ejemplo, en relación con el intercambio de conocimientos, pero también muchos de los colectivos señalan que los prototipos realizados durante el laboratorio les han sido útiles para expandir su quehacer tanto individual como colectivo.

\section{Sostenibilidad institucional}

La sostenibilidad institucional de los procesos comunitarios está condicionada por factores externos e internos que inciden a favor o en contra. Entre ellos está la existencia de políticas públicas que soporten las prácticas y proyectos de las organizaciones en cuestión. Es también importante en esa dimensión que los medios y recursos requieren que los procesos sean suficientes y puedan gozar de autonomía para su uso. Adicionalmente, se encuentra el tejido de relaciones que se construye al interior y al exterior del proceso, de las que hacen parte el establecimiento de relaciones con otras organizaciones e instituciones.

Este componente de la sostenibilidad es, quizá, el que más ha impactado el laboratorio. En primer lugar, porque desde su propuesta inicial el ComunLAB ha querido facilitar la interlocución entre la institucionalidad y los colectivos, cuyas relaciones han sido distantes $y$, en algunos casos, problemáticas. Ha ocurrido entonces que se facilita la comunicación entre ambos, permitiendo que el encuentro se libere un poco de la formalidad que ha caracterizado los diálogos existentes con algunas instituciones. Para algunos de los colectivos, el laboratorio impulsó dinámicas de largo aliento, como permitirles...

Fortalecerse en ese sector público, político, de saber que si digamos nos unimos y fortalecemos, pues de alguna manera podemos transformar algo político y social dentro de la comunidad. El ComunLAB puede incidir de alguna forma en la construcción de una política pública (Colectivo Satélite Sursystem, 2013). 
Otro componente importante de la sostenibilidad institucional que ha creado el laboratorio tiene que ver con la creación de redes, tanto entre los colectivos como con las instituciones y otras organizaciones. De esta forma se propicia, tal como lo sugiere Mestres (2012, p.43), el "elaborar desde los diferentes lugares-mentes que cooperan 'procesos propios' desvinculados de las lógicas de la pleitesía a las que obliga el paradigma 'cooperador-cooperado'" en los que caen muchos de los proyectos que buscan apoyar iniciativas u organizaciones de base. Sin duda, las redes entre los colectivos siempre han existido y forman parte de la estrategia que ellos implementan para su sostenibilidad. Es importante destacar que el ComunLAB no las crea, las potencia.

Otro elemento que es transversal al laboratorio y que parece resultar significativo para esta dimensión de la sostenibilidad tiene que ver con propiciar espacios para la creación colectiva y el encuentro entre diferentes colectivos, de los que se derivan la generación de redes y la activación de proyectos conjuntos.

\section{Sostenibilidad económica}

Retomando a Mestres (2012, p. 30), se reconoce que:

El valor económico y el valor cultural no son necesariamente antitéticos; es deseable lograr un equilibrio entre ambos criterios de análisis. El fracaso económico no es sinónimo de calidad cultural, ni el éxito económico coincide con la ausencia de esta calidad [...] la introducción de una mirada económica sobre la realidad cultural no pone en crisis el concepto de rentabilidad cultural como criterio fundamental.

En las organizaciones comunitarias la sostenibilidad económica depende en muchos casos de la gestión que hagan de las otras dos dimensiones de la sostenibilidad, en tanto su capital social coadyuva a la realización de sus prácticas. La gestión efectiva de los recursos favorece el logro de los objetivos de los colectivos. En este campo, los modelos "son quizá distintos en la mayoría de los casos, aunque no las necesidades, y el escenario en el que todos estos agentes independientes han de luchar por conseguir sus propios fines" (Mestres, 2012, p.31).

Si bien el ComunLAB no ha impactado de manera sustancial en la sostenibilidad económica de los colectivos, la articulación de otros elementos incentivados dentro del laboratorio, como la generación de redes y el fortalecimiento de su sostenibilidad social, ha llegado, en algunos casos, a estimular su sostenibilidad económica. 
Igualmente, las alianzas y articulación del laboratorio con algunas instituciones han permitido que estas conozcan los procesos de los colectivos e, incluso, que de ello resulten apoyos económicos para enriquecer las actividades del laboratorio en las que han participado los colectivos. Es recurrente que ellos reconozcan que el laboratorio se ha preocupado por valorar su trabajo y, en la medida en que ha sido posible, que exista una contraprestación para aquellos que comparten sus saberes en los intercambios: "nos ha dejado trabajar. Nos ha generado recursos, que en muchas ocasiones [las carencias] nos han tenido un poco maniatados" (Colectivo Dektol, 2013).

\section{Conclusiones}

El estudio de caso realizado sobre el ComunLAB permitió identificar estrategias que se ponen en marcha en los procesos locales de diseño social. A propósito de los procesos de diseño social en lo local, el caso permite dilucidar que dichas estrategias no se ponen en uso únicamente por el hecho de exigirle a un proyecto que atienda a los lineamientos de este tipo de enfoque; sino que es el mismo problema, las necesidades del proyecto y los actores involucrados lo que genera la aplicación de ciertas tácticas, en lugar de otras. Y eso precisamente conecta el caso al diseño social y permite, además, un acercamiento a esta categorización de las formas de diseñar que engloba el enfoque en mención.

Preguntarse por las estrategias y analizarlas dentro de este caso, abre la pregunta sobre qué es lo que le otorga a tal o cual práctica de diseño su carácter social. Desde estos hallazgos es claro que para pensar en ello son claves las metodologías, las técnicas y, por supuesto, las estrategias que se utilizan para analizar un problema y emprender sobre ello un proceso de diseño localizado. 
De otro lado, es importante mencionar que la existencia y uso de estrategias no pueden ser entendidas como definitivas, susceptibles de estandarizarse y asegurar que aplicándolas se llegará inequívocamente a un diseño social. Queda claro que son guías que orientan a la acción y que uno de los grandes potenciales y aciertos de un diseño social es su posibilidad de leer los contextos. Lo que le permite reconfigurar, adaptar y resignificar dichas estrategias para asegurar su efectividad en las realidades en las que se desarrollan.

Después de haber identificado las estrategias presentes en ComunLAB, resultan claves los siguientes tres elementos que identifica Mestres (2012, p.137) para el desarrollo de procesos en y con comunidad:

Confianza y respeto mutuo: Si no la hay, difícilmente puede darse un proceso honesto de colaboración, enriquecedor para todas las personas que participen y/o sean afectadas por el mismo.

Reciprocidad: Estar dispuestos a compartir y corresponder de igual a igual, a dar y recibir, a aprender con y del otro.

Relevancia: Reconocimiento justo de las contribuciones de cada persona, teniendo en cuenta que, aunque todo el mundo no vaya a contribuir por igual, cada cual debe tener la posibilidad de contribuir de manera clave.

Respecto a la incidencia que ha tenido el laboratorio en los colectivos durante sus cuatro años de trayectoria, es preciso leer al ComunLAB como una plataforma que propicia el encuentro, que activa las redes que ya existen entre los colectivos y estimula la creación colectiva.

En este sentido, los aportes del ComunLAB se suman a los generados por una serie de iniciativas de base, más de carácter autónomo y autogestionado en la cotidianidad. De esta manera, es claro que el ComunLAB no garantiza la sostenibilidad de los colectivos, pero sí ha buscado pensar su accionar a favor de potenciar los procesos y ejercicios que estos han realizado para hacerse más sostenibles. 
Lo anterior ha arrojado resultados en las tres dimensiones de la sostenibilidad: social, institucional y económica, como las que se abordaron a lo largo del "análisis profundo". Entonces, el laboratorio ha permitido reivindicar y legitimar las prácticas colectivas de larga data, en busca de hacerlas visibles ante actores e instituciones con quienes se han tenido acercamientos conflictivos o, incluso, nulos. $\mathrm{Y}$, de algún modo, pretende servir de mediador entre estas relaciones.

Por último, a propósito de los beneficios que el laboratorio ha generado en términos de sostenibilidad, es importante, de antemano, pensar que procesos como estos no proporcionan soluciones determinantes a problemáticas complejas que dependen de diversos factores, como es el caso de la sostenibilidad de los colectivos. No solo porque ningún diseño de este tipo logra este cometido, sino porque no es un objetivo al que aspire o se comprometa desde los sentidos y prácticas que alienta; y esto es algo que aplica para cualquier proceso de diseño social.

\section{REFERENCIAS BIBLIOGRÁFICAS}

Aguirre, J. (2018). Pistas para aproximarse al diseño social: antecedentes y posturas. Kepes, (17), 9-27.

Aguirre, J. \& Cifuentes, M. (2018). El diseño es otro cuando actúa en el contexto social. En: Memorias del VII simposio Internacional de Diseño Social (20 y 21 de septiembre, Medellín, 2018), 92-99.

Armstrong, L., Bailey, J., Julier, G., \& Kimbell, L. (2014). Social Design Futures: HEI Research and the AHRC. Londres: University of Brighton and Victoria and Albert Museum.

Cross, N. (1999). Métodos de diseño: estrategias para el diseño de productos. México D.F.: Noriega Editories. 
Chui, M., Manyika, J., Bughin, J., Dobbs, R., Roxburgh, C., Sarrazin, H., Sands, G.\& Westergren, M. (2012). The social economy: Unlocking value and productivity through social technologies. McKinsey Global Institute. Recuperado el 1 de abril de 2015, de: http://www.mckinsey.com/industries/high-tech/ our-insights/the-social-economy

Gumucio, A. (2005). Arte de equilibristas: la sostenibilidad de los medios de comunicación comunitarios. Punto Cero, 10(10), 6-19.

Holston, D. (2011). The Strategic Designer: Tools \& techniques for managing the design process. Cincinnati, USA: How Books.

Mestres, A., Guido, T., Lapuente, E. (2012). Música para camaleones: el black álbum de la sostenibilidad cultural. Barcelona: Tránsit Projectes.

Meroni, A. (2008). Strategic design: where are we now? Reflection around the foundations of a recent discipline. Strategic Design Research Journal, 1(1), 31-28.

Sanders, E. (2006). Design research in 2006. Design Research Quarterly. Design Research Society (1) September, 3-8.

Sanders, L., \& Simons, G. (2009). A social vision for value co-creation in design. Open Source Business Resource, (December), 27-34.

Sanders, E. , \& Stappers, P. J. (2008). Co-creation and the new landscapes of design. Co-design, 4(1), 5-18. 